\title{
NEW RESULTS ON THE NOISE FIGURE OF HEMTs
}

\author{
M.A. GRADO CAFFARO \\ consultant, Madrid, Spain \\ M. GRADO CAFFARO \\ CSIC, Madrid, Spain
}

(Received March 3, 1993; in final form March 31, 1993)

In this communication, an accurate mathematical model for the noise figure of a high electron mobility transistor is developed. This model represents a substantial improvement of the Fukui model. In fact, the Fukui approach can be considered as an approximation of our model under certain conditions.

The fundamental relationship that gives minimum noise figure is derived from a first-order linear partial differential equation, which is established by assuming reasonable statements based on experience.

\section{INTRODUCTION}

It is well-known that minimum noise figure of HEMTs depends strongly on frequency and the important parasitic parameters: gate and source resistances, and gate-source capacitance ${ }^{1}$. The values of these elements and the frequency can be considered as real variables so that noise figure can be expressed as a function depending on these variables; by virtue of Fukui approach this function depends linearly on the frequency for a given device ${ }^{2,3}$.

In the following, we shall state a new formulation based on the properties of certain linear partial differential equations in order to generalize the mathematical formulation of HEMT's noise figure.

\section{NOISE FIGURE MODELISATION}

First, minimum noise figure can be expressed as follows ${ }^{2.4}$ :

$\mathrm{F}_{\min }=1+\mathrm{mf}$

where $f$ is the frequency and $m$ is proportional to $C_{g s}\left(R_{s}+R_{g}\right)^{1 / 2} \cdot g_{m}^{-1 / 2}$; here $C_{g s}$ is the gate-source capacitance, and $R_{s}$ and $R_{g}$ are the source and gate resistances, respectively; $\mathrm{g}_{\mathrm{m}}$ denotes transconductance.

It is possible to establish a partial differential equation whose complete solution coincides with the Eqn. (1). In order to obtain this equation, we note that the 
relative variation of $F_{\min }$ is proportional to the relative variation of each variable in Eqn. (1); we define relative variation of a magnitude as the ratio: variation of this magnitude/value of it. From experimental data, we can claim that the proportionality "constant" involved in the above assumption is the same for the two variables. Therefore we have:

$\frac{\Delta F_{\min }}{F_{\min }}=A \cdot \frac{\Delta m}{m}$

$\frac{\Delta F_{\min }}{F_{\min }}=A \cdot \frac{\Delta f}{f}$

(with A depending on $\mathrm{m}$ and $\mathrm{f}$ ) where $\Delta$ denotes variation (absolute variation), and $A$ is the pseudoconstant mentioned above. Since $\lim _{\Delta \mathrm{m} \rightarrow 0}\left(\Delta \mathrm{F}_{\min } / \Delta \mathrm{m}\right)=\partial \mathrm{F}_{\min } /$ $\partial \mathrm{m}$ and $\lim _{\Delta t \rightarrow 01}\left(\Delta \mathrm{F}_{\min } / \Delta \mathrm{f}\right)=\partial \mathrm{F}_{\min } / \partial \mathrm{f}$. From (2) and (3) we obtain

$\mathrm{m} \frac{\partial \mathrm{F}_{\min }}{\partial \mathrm{m}}=\mathrm{A}(\mathrm{m}, \mathrm{f})$

and

$f \frac{\partial F_{\min }}{\partial f}=A(m, f)$

and hence

$\mathrm{m} \frac{\partial \mathrm{F}_{\min }}{\partial \mathrm{m}}=\mathrm{f} \frac{\partial \mathrm{F}_{\min }}{\partial \mathrm{f}}$

Next, we will solve the first-order linear partial differential equation (6). To get this end, we formulate the differential system associated with Eq. (6) namely

$\frac{\mathrm{dm}}{\mathrm{m}}=-\frac{\mathrm{df}}{\mathrm{f}}=\frac{\mathrm{dF}_{\min }}{0}$

whose solution is

$$
\left.\begin{array}{rl}
\mathrm{mf} & =\mathrm{C}_{1} \\
\mathrm{~F}_{\min } & =\mathrm{C}_{2}
\end{array}\right\}
$$

where $\mathrm{C}_{1}$ and $\mathrm{C}_{2}$ are constants.

Therefore, the general solution of Eq. (6) is given by

$\Phi\left(\mathrm{C}_{1}, \mathrm{C}_{2}\right)=0$ or $\Phi\left(\mathrm{mf}, \mathrm{F}_{\min }\right)=0$ 
On the other hand, a complete solution to Eq. (6) can be expressed as follows ${ }^{5}$ :

$\mathrm{v}=\mathrm{k}_{1} \mathrm{u}+\mathrm{k}_{2}$

where $k_{1}$ and $k_{2}$ are constants, and $u$ and $v$ depend on $m, f$, and $F_{\min }$ so that $u(m$, $\left.\mathrm{f}, \mathrm{F}_{\text {min }}\right)=\mathrm{C}_{1}$ and $\mathrm{v}\left(\mathrm{m}, \mathrm{f}, \mathrm{F}_{\min }\right)=\mathrm{C}_{2}$. Then, by considering (8), (9) and (10) we have

$\mathrm{F}_{\min }=\mathrm{k}_{1} \mathrm{mf}+\mathrm{k}_{2}$

Now, we compute $\mathrm{k}_{2}$ by assuming that $\mathrm{F}_{\min }=1$ when $\mathrm{mf}=0$; hence $\mathrm{k}_{2}=1$. Moreover, when $\mathrm{mf}=1$ we have $\mathrm{F}_{\min }=2$ and consequently $\mathrm{k}_{1}=1$. Then, equation (11) reduces to $F_{\min }=1+\mathrm{mf}$, that is the relationship (1).

The crucial point of our research work is related to the fact that the complete integral of Eq. (6) is not unique. In fact, another complete solution is

$F_{\min }=e^{m f}$

In effect, it is easy to prove that Eq. (12) satisfies Eq. (6). The Mc. Laurin expansion of $(12)$ is

$F_{\min }=\sum_{n=0}^{\infty} \frac{(m f)^{n}}{n !}$

When $\mathrm{mf} \ll 1$, Eq. (13) can be reduced to

$\mathrm{F}_{\min } \cong 1+\mathrm{mf}$

There exist infinite complete solutions to Eq. (6). These solutions obey Eq. (9), and Eq. (11) represents the standard complete integral (affine function in $\mathrm{mf}$ ). Moreover, all the terms in the right-hand side of the expression (13) satisfy Eq. (6) and, since this equation is linear, the sum of these infinite terms converges to a function that satisfies Eq. (6); this function is given by Eq. (12). Therefore, it is clear that, in our context, the "best" solution is Eq. (12).

In addition, it is easy to see that the relationship (12) represents a minimum noise figure. By applying variational techniques to an adequate formula to describe noise, it is possible to get the previous result.

\section{DISCUSSION}

It is evident that the model developed here improves substantially the noise formulation of Fukui which is given usually by $F_{\min }(d B)=10 \log _{10}(1+\mathrm{mf})$. When the inequality $\mathrm{mf} \ll 1$ does not occur, the Fukui model is inadequate and Eq. (12) is really suitable for a typical $\mathrm{GaAs} / \mathrm{AlGaAs} \mathrm{HEMT}$ operating at frequencies greater than $5 \mathrm{GHz}$ with $\mathrm{m} \cong 0.02 \mathrm{~ns}$. 
When we assume that $\mathrm{A}$ does not depend approximately on $\mathrm{m}$ and $\mathrm{f}$, it is easy to see that $F_{\min } \cong A \ln (m f)+C$ where $C$ is a constant, that is, $C$ depends on $A$, and does not depend on $\mathrm{m}$ and $\mathrm{f}$. The above expression is incorrect because it cannot be proven experimentally. Then, we conclude that the dependence on $\mathrm{m}$ and $\mathrm{f}$ is considerable. Eq. (12) is in good agreement with experimental work ${ }^{6}$ and, therefore, it represents a full solution to the noise problem with respect to high electron mobility transistors.

On the other hand, the following fact is remarkable: in order to determine the constant $\mathrm{k}_{1}$ in Eq. (11), $\mathrm{F}_{\min }=2$ so that $\mathrm{F}_{\min }=2(=3 \mathrm{~dB})$ corresponds to $\mathrm{f}=$ $1 / \mathrm{m}$ (cutoff frequency with respect to noise figure).

\section{REFERENCES}

1. R.J. Hamilton, Jr. and S.G. Bandy, "HEMT-Based MMIC Amplifier for Enhanced EW System Performance." Microwave Journal, vol. 31, no. 2, pp. 167-174 (1988).

2. H. Fukui, “Optimal noise figure of microwave GaAs MESFETs." IEEE Trans. Electron Devices, vol. ED-26. pp. 1032-1037 (1979).

3. R.A. Pucel, H.A. Haus and H. Statz, "Signal and noise properties of GaAs microwave field effect transistors," in Advances in Electronics and Electron Physics; L. Marton, ed., vol. 38, New York, Academic Press, 1975, p. 195.

4. M.A. Grado and M. Grado, "Noise minimization in microwave amplifiers." Eurofach Elect., vol. 173. pp. 52-54 (1990).

5. P.W. Berg and J.L. McGregor, "Elementary partial differential equations," Holden-Day, San Francisco, 1966.

6. S.S. Pei, N. J. Shah, R.H. Hendel, C.W. Tu, M.D. Feuer and R. Dingle. IEEE GaAs IC Symp., Digest of Tech. Papers, pp. 129-132; IEEE, New York (1984). 

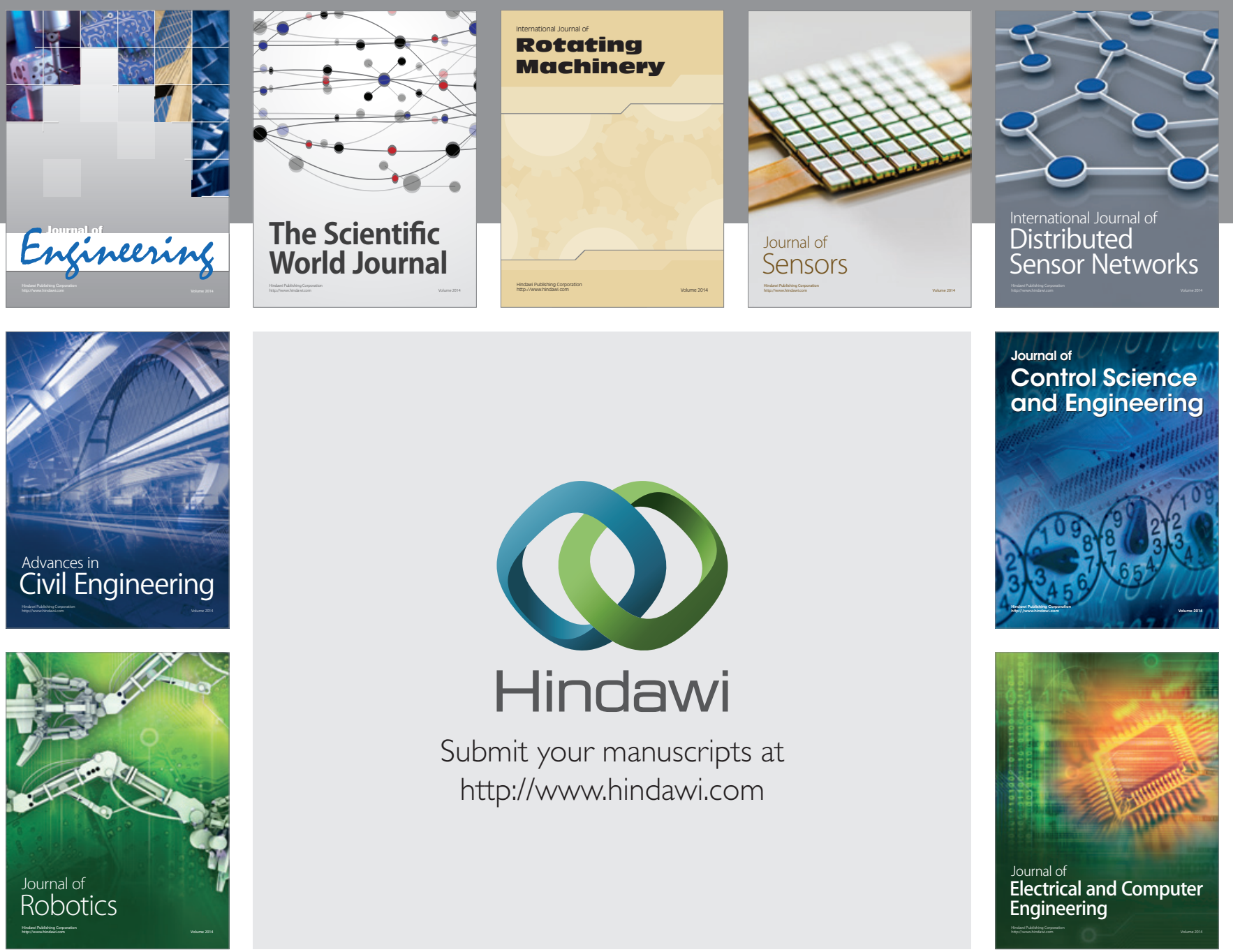

Submit your manuscripts at

http://www.hindawi.com
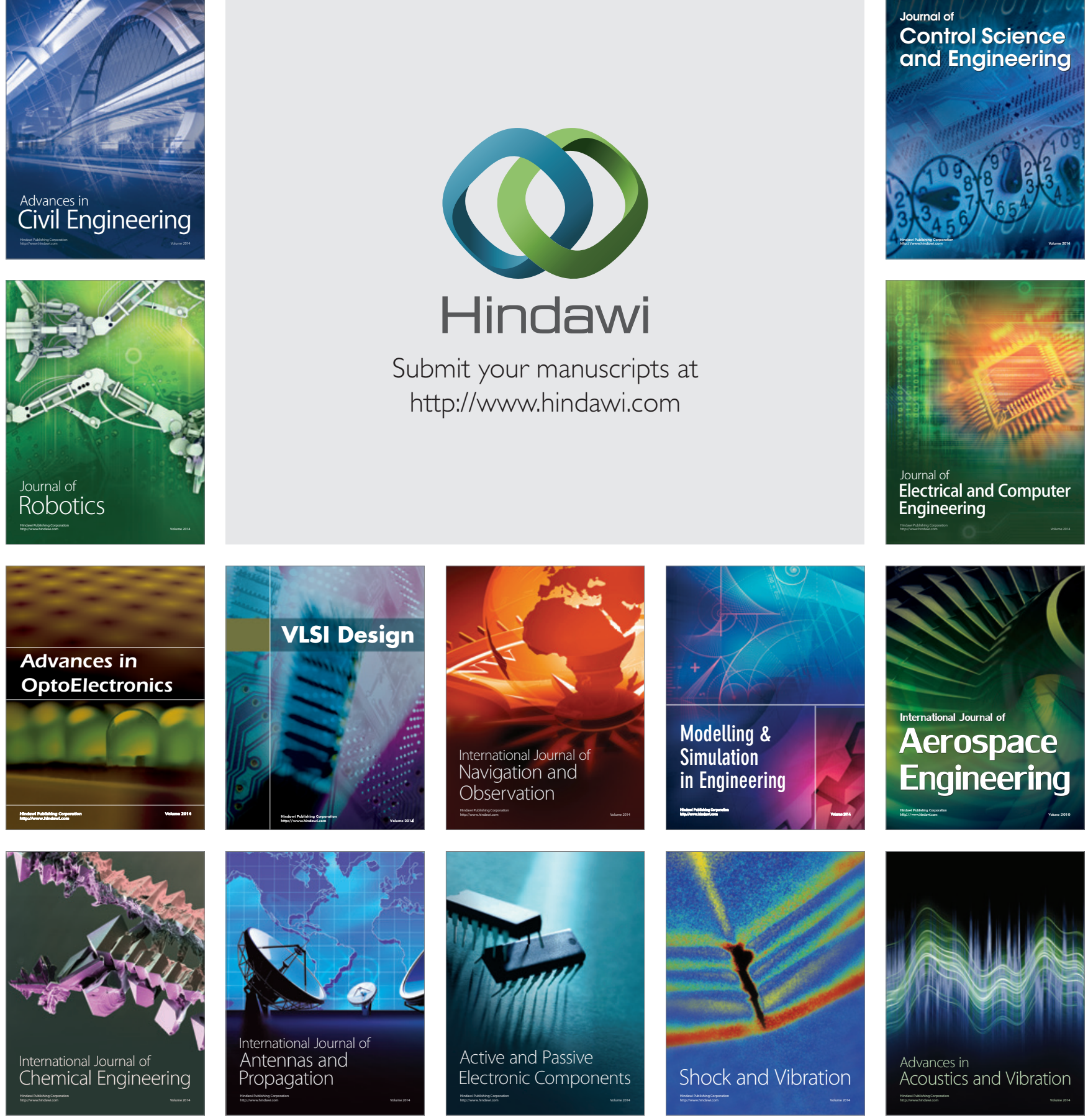